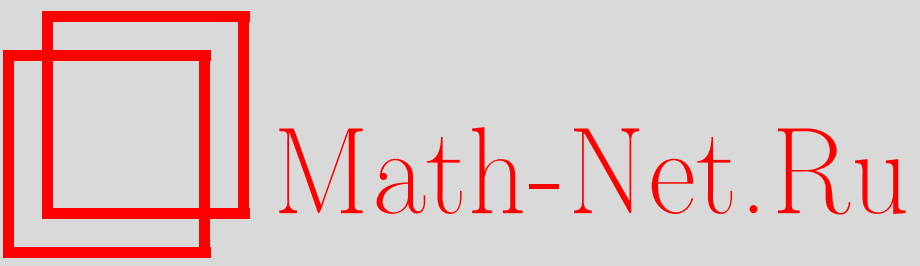

Е. И. Бунина, Элементарная эквивалентность групп Шевалле, УМН, 2001, том 56, выпуск 1, 157-158

DOI: https://doi.org/10.4213/rm362

Использование Общероссийского математического портала Math-Net.Ru подразумевает, что вы прочитали и согласны с пользовательским соглашением

http://www.mathnet.ru/rus/agreement

Параметры загрузки:

IP: 18.234 .156 .22

26 апреля 2023 г., 16:58:53 


\title{
ЭЛЕМЕНТАРНАЯ ЭКВИВАЛЕНТНОСТЬ ГРУПП ШЕВАЛЛЕ
}

\author{
Е. И. БунинА
}

ОПРЕДЕЛЕНИЕ. Элементарными свойствами алгебраической системы называются те свойства этой системы, которые можно выразить на языке узкого исчисления предикатов (УИП), принимая в качестве исходных основные операции и отношения данной системы. Две алгебраических системы одинаковой сигнатуры назьваются әлементарно әквивалентными (обозначение: $G_{1} \equiv G_{2}$ ), если они обладают одинаковыми элементарными свойствами.

Вопросы элементарных свойств линейных групп были впервые рассмотрены в 1961 году А.И. Мальцевым. В работе "Об элементарных свойствах линейных групп" [1] он доказал следуюшую структурную теорему: для того чтобь группы $\mathscr{G}\left(m, K_{1}\right)$ u $\mathscr{G}\left(n, K_{2}\right)(\mathscr{G}=G L$, $P G, S L, P S L, m \geqslant n \geqslant 3)$ имели один и тот же арифметический тип (были әлементарно әквивалентны), необходимо и достаточно, чтобь $m=n$ и чтобы один и тот же арифметический тип имели поля $K_{1}$ и $K_{2}$.

Продолжение эта теория получила в 1992 году, когда прогресс в теории моделей позволил К.И. Бейдару и А.В. Михалеву предложить в работе [2] общий способ решения проблем такого типа. С помощью логических конструкций они обобщили теорему Малшцева для случая групп $G L, S L, P G, P S L$ над первичными ассоциативными кольцами, содержащими 1 и $1 / 2$, и над телами. Кроме того, ими была доказана теорема, позволяющая выводить утверждения об элементарной эквивалентности алгебраических систем из утверждений об их изоморфности.

Группы Шевалле, рассматриваемые в данной работе, включают в себя такие классические группы, как $S L_{n}(K), P S L_{n}(K), S O_{n}(K), \operatorname{Spin}_{n}(K), P S O_{n}(K), S p_{2 n}(K), P S p_{2 n}(K)$, т.е., с одной стороны, изучаемые группы пересекаются с теми, которые рассматривались А. И. Мальцевым в работе [1], с другой стороны, здесь присутствует доволшно много классических линейных групп, им не рассмотренных.

Основным резултатом работы является следующая теорема.

Теорема. Предположим, что группы Шевалле $\mathscr{G}_{1}$ и $\mathscr{G}_{2}$ построены по алгебраически замкнутым полям $k_{1}$ и $k_{2}$ характеристик, не равных двум, простым алгебрам Ли $\mathscr{L}_{1}$ и $\mathscr{L}_{2}$ и решеткам $L$ и $M$. Пусть, кроме того, $L / L_{0} \cong \varphi_{1}, M / M_{0} \cong \varphi_{2}$, где $\varphi_{1}$ и $\varphi_{2}$ - конечные группь. Тогда $\mathscr{G}_{1} \equiv \mathscr{G}_{2}$ тогда и только тогда, когда $k_{1} \equiv k_{2}, \mathscr{L}_{1} \cong \mathscr{L}_{2} u$ $\varphi_{1} \cong \varphi_{2}$.

СХЕМА ДОКАЗАТЕЛЬСТВА. Предположим сначала, что группы Шевалле $\mathscr{G}_{1}$ и $\mathscr{G}_{2}$ построены по одной и той же алгебре Ли $\mathscr{L}$ и фундаментальной группе $\varphi$ и по элементарно эквивалентным полям $k_{1}$ и $k_{2}$. Каждое элементарно групповое предложение $\mathscr{U}$, относящееся к группам Шевалле $\mathscr{G}_{1}$ и $\mathscr{G}_{2}$, можно преобразовать в кольцевую формулу $\mathcal{U}^{k}$, относящуюся к полям $k_{1}$ и $k_{2}$, так, чтобы истинность $\mathscr{U}$ на $\mathscr{G}$ была равносильна истинности $\mathscr{U}^{k}$ на $k$. Вид формулы $\mathscr{U}^{k}$ при этом не будет зависеть от поля $k$. Таким образом, истинность $\mathscr{U}$ на $\mathscr{G}_{1}$ равносильна истинности $\mathscr{U}^{k}$ на $k_{1}$. Если поля $k_{1}$ и $k_{2}$ элементарно эквивалентны, то истинность $\mathscr{U}^{k}$ на $k_{1}$ равносильна истинности $\mathscr{U}^{k}$ на $k_{2}$. Поэтому истинность $\mathscr{U}$ на $\mathscr{G}_{1}$ равносильна истинности $\mathscr{U}$ на $\mathscr{G}_{2}$, следовательно, $\mathscr{G}_{1}$ и $\mathscr{G}_{2}$ элементарно эквивалентны.

Теперь предположим, что группы $\mathscr{G}_{1}$ и $\mathscr{G}_{2}$ элементарно эквивалентны. Известно, что фундаментальная группа группы Шевалле $\mathscr{G}$ совпадает с ее центром, поэтому группы $\varphi_{1}$ и $\varphi_{2}$ изоморфны. Профакторизуем группы $\mathscr{G}_{1}$ и $\mathscr{G}_{2}$ по их центрам, получим элементарно эквивалентные группы $\overline{\mathscr{G}}_{1}$ и $\overline{\mathscr{G}}_{2}$.

Предположим, что группа Шевалле $\mathscr{G}$ построена по алгебре Ли типа $A_{n}, B_{n}, C_{n}, D_{n}$ или $G_{2}$. Тогда предложение

$$
D d_{r}(X, M):=\left(X M=M X \& M^{2^{r}}=1 \&((Y)(Z)(Y M=M Y \& Z M=M Z \rightarrow Z Y=Y Z))\right)
$$

для фиксированной матрицы $M$ при некотором $r$ выделяет такие $X \in \mathscr{G}$, что они составляют подгруппу матриц, диагонализируемых в одном базисе (обозначим ее через $\mathscr{H}$ ). При этом 
$N(\mathscr{H}) / \mathscr{H} \simeq W$, где $W$ - группа Вейля соответствующей алгебры Ли $\mathscr{L}$. Так как группы Вейля алгебр Ли $A_{n}, B_{m}\left(C_{m}\right), D_{l}$ и $G_{2}$ для всех $n \geqslant 1, m \geqslant 2, l \geqslant 3$ неизоморфны, то для каждой системы корней указанного вида существует элементарно групповое предложение, истинное в группах Шевалле, у которых алгебра Ли имеет данную систему корней, и ложное в группах Шевалле с любой другой алгеброй Ли. Группы Шевалле с системами корней $B_{n}$ и $C_{n}$ не могут быть элементарно эквивалентны, так как имеют разное количество классов инволюций. Теперь рассмотрим произвольную группу Шевалле $\mathscr{G}$ указанного в теореме вида и проверим, сколько в ней есть классов инволюций. Если их количество равно 1 или больше 3 , то перед нами некоторая группа типа, рассмотренного выше, для которого мы умеем определять ее систему корней. Если в группе два класса инволюций, то это либо классическая группа, либо группа типа $F_{4}, E_{6}$ или $E_{8}$. Рассмотрим для каждой инволюции в каждой из таких групп ее централизатор, а затем коммутант этого централизатора. Полученные коммутанты будут изоморфны уже рассмотренным классическим группам Шевалле или их центральным произведениям. Найдем для каждой из систем корней $F_{4}, E_{6}$ и $E_{8}$ такое предложение, что оно истинно на коммутанте централизатора одной из инволюций в каждой группе с соответствующей системой корней ее алгебры Ли и ложно на коммутанте централизатора каждой инволюции в любой другой группе Шевалле с двумя классами инволюций. С помощью этого предложения мы построим элементарно групповое предложение, отличающее группы типов $F_{4}, E_{6}, E_{8}$ друг от друга и от остальных групп Шевалле. Аналогичным способом строится предложение, отличающее группу Шевалле типа $E_{7}$, но для этого рассматриваются группы с тремя классами инволюций. Таким образом, мы получаем, что если группы $\overline{\mathscr{G}}_{1}$ и $\overline{\mathscr{G}}_{2}$ элементарно эквивалентны, то их алгебры Ли изоморфны.

Теперь для каждого типа алгебры Ли доказывается, что каждое элементарно кольцевое предложение $\mathscr{U}$, относящееся к полям $k_{1}$ и $k_{2}$, можно преобразовать в элементарно групповое предложение $\mathscr{U}_{\mathscr{G}}$, относящееся к группам Шевалле $\overline{\mathscr{G}}_{1}$ и $\overline{\mathscr{G}}_{2}$ данного типа и равносильное первоначальному (доказательство проводится для каждого типа алгебры Ли; в случае классических групп Ли мы выделяем предложения, которым удовлетворяют те и только те матрицы, которые вместе со специально введенными операциями "сложения" и "умножения" становятся изоморфными полю $k$; в случаях исключительных алгебр Ли мы рассматриваем коммутанты централизаторов инволюций, в них выделяем относительно элементарные подгруппы, изоморфные классическим, а затем ссылаемся на предыдущие случаи). Так как вид предложения $\mathscr{U}_{G}$ одинаков для групп $\overline{\mathscr{G}}_{1}$ и $\overline{\mathscr{G}}_{2}$ и истинность $\mathscr{U}_{G}$ на $\overline{\mathscr{G}}_{1}$ равносильна истинности $\mathscr{U}_{G}$ на $\overline{\mathscr{G}}_{2}$, то истинность $\mathscr{U}$ на $k_{1}$ равносильна истинности $\mathscr{U}$ на $k_{2}$, т.е. поля $k_{1}$ и $k_{2}$ элементарно эквивалентны.

Теорема доказана полностью.

\section{СПИСОК ЛИТЕРАТУРЫ}

[1] А. И. Мальцев // Проблемы математики и механики. Новосибирск: Изд-во АН СССР, 1961. C. 110-132. [2] C. I. Beidar, A. V. Michalev // Contemp. Math. 1992. V. 131. P. 29-35.

Московский государственньй университет им. М.В. Ломоносова
Принято редколлегией

01.12 .2000 